\title{
Edge Stability and Pedestal Profile Sensitivity of Snowflake Diverted Equilibria in the TCV Tokamak
}

\author{
S. Yu. Medvedev*1, A. A.Ivanov ${ }^{1}$, A. A. Martynov ${ }^{1}$, Yu. Yu. Poshekhonov ${ }^{1}$, R. Behn ${ }^{2}$, Y. R. \\ Martin $^{2}$, J-M. Moret ${ }^{2}$, F. Piras ${ }^{2}$, A. Pitzschke ${ }^{2}$, A. Pochelon ${ }^{2}$, O. Sauter ${ }^{2}$, and L. Villard ${ }^{2}$ \\ ${ }^{1}$ Keldysh Institute, Russian Academy of Sciences, Moscow, Russia \\ ${ }^{2}$ Ecole Polytechnique Fédérale de Lausanne (EPFL), Centre de Recherches en Physique des Plasmas (CRPP), \\ Association Euratom-Confédération Suisse, Lausanne, Switzerland
}

Received 31 August 2009, revised 12 October 2009, accepted 15 October 2009

Published online 10 May 2010

Key words Kink-ballooning modes, pedestal profiles, snowflake divertor.

A second order null divertor (snowflake) has been successfully created and controlled in the TCV tokamak [1] (F. Piras et al. , Plasma Phys. Control. Fusion, 2009). The results of ideal MHD edge stability computations show an enhancement of the edge stability properties of the snowflake equilibria compared to standard x-point configurations [2] (S. Yu. Medvedev et al. , 36th EPS Conference on Plasma Physics, 2009). However, a sensitivity study of the stability limits to variations of the pedestal profiles is essential for making conclusions about possibilities of ELM control in snowflake plasmas. Variations of the edge stability and beta limits for several types of snowflake equilibria, different values of triangularity and various pedestal profiles are investigated.

(c) 2010 WILEY-VCH Verlag GmbH \& Co. KGaA, Weinheim

\section{Introduction}

The snowflake (SF) divertor configuration was proposed in [3]. The second order null instead of the x-point in a standard single null (SN) configuration modifies the magnetic topology near the plasma boundary and is therefore expected to affect the edge plasma properties. In particular, the flux expansion around the null point is 2-3 times larger than in the SN configuration and the connection length in that region increases, reducing the local heat load to the divertor plates [4]. The magnetic shear in the edge where an H-mode pedestal would lie is modified, providing a possible way to influence edge localized mode (ELM) activity.

The MHD stability of the plasma edge provides a good guide to ELM triggering conditions in tokamak [5]. A systematic investigation of the edge stability in tokamak should also include pedestal profile sensitivity studies. As shown in [6] the ratio of the edge to maximum values of the current density and pressure gradient in the pedestal is an important parameter for the sensitivity study. In the present paper the edge stability of the SF and SN plasmas is compared and new scalings for the pedestal poloidal beta $\beta_{p, p e d}$ are proposed taking into account the variations of the pedestal profiles.

\section{Equilibrium and stability of snowflake configurations}

\subsection{Equilibrium}

The free-boundary equilibrium code SPIDER (reconstruction mode) has been modified to compute equilibria with a snowflake divertor [7]. An option to maintain the second order null in a prescribed position was added to the standard prescription of "limiter" points at the plasma boundary and a set of control points approximately specifying the target plasma shape while minimizing the sum of squared values of the coil currents. The null points and the separatrix are assumed to be always in vacuum with the plasma boundary defined by a prescribed fraction of poloidal flux inside the separatrix. Let us note that, in addition to vanishing first derivatives it is sufficient to impose two conditions on the second derivatives at the second order null: $\partial^{2} \psi / \partial Z^{2}=0$ and $\partial^{2} \psi / \partial R \partial Z=0$.

* Corresponding author: e-mail: medvedev@a5.kiam.ru; Phone: +7 4999781314 Fax: +7 4999720737 
Then the third one, $\partial^{2} \psi / \partial R^{2}=0$, is satisfied due to $\Delta^{*} \psi=0$ for the poloidal flux function $\psi$ in vacuum, where $\Delta^{*}=R(\partial / \partial R)(1 / R)(\partial / \partial R)+\partial^{2} / \partial Z^{2}$ is the Grad-Shafranov operator. Using the reconstructed boundary for the TCV snowflake shot \#36151 as a target shape (but not the profiles), a variety of free boundary equilibria with different profiles and positions of the snowflake point has been obtained (Fig.1). The plasma current $I_{p}=300 k A$ was prescribed for all cases.

The values of the poloidal field coils currents for creating the SF plasmas in TCV are rather high compared to the usual SN configurations [1]. In the SPIDER equilibrium calculation no attempt was made to optimize the values of the currents which are subject to several engineering constraints. All the calculations were performed using plasma profiles close to those measured in TCV single null $\mathrm{H}$-mode plasmas [8]. The flexibility of the TCV magnetic system for creating the SF equilibria with different locations of the second order null point (close to the inner wall in Fig.1c) and positions of plasma in the vacuum chamber (at the bottom in Fig.1d) was demonstrated. The SN equilibria with the x-point in the place of the second order null were computed using the same set of control points. The plasma boundaries for SF and SN equilibria are close to each other at the low field side (Fig.1e, solid lines). The equilibria with increased triangularity were obtained by adding a "limiter" point at a suitable position (Fig.1b).

A specific feature of free boundary snowflake equilibria is a significant sensitivity of the plasma shape in the vicinity of the snowflake point to the current density in the pedestal. In Fig.1e the plasma boundary (dashed line) for quadruple values of the pedestal current density and pressure gradient is compared to the SF and SN boundaries (the same set of control points was used). The plasma boundary deformation leads to a further increase of the shear thus preventing its reversal even for very high values of pedestal current density.

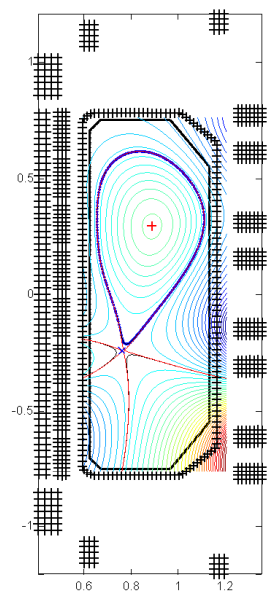

a)

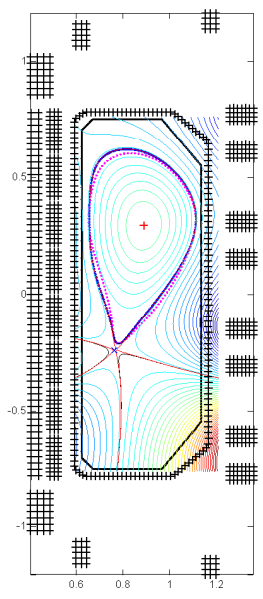

b)

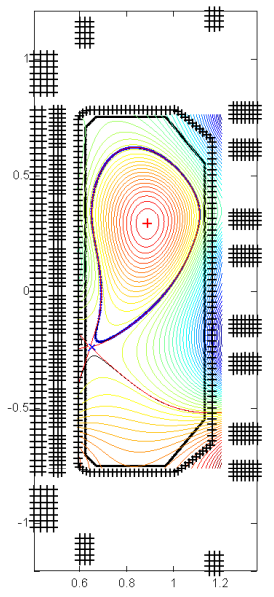

c)

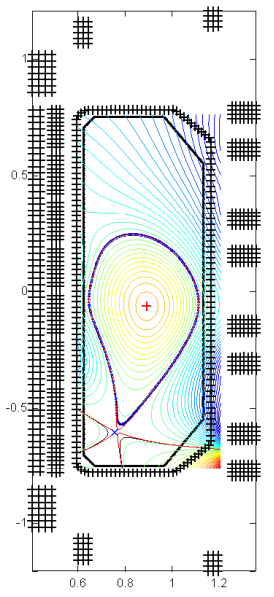

d)

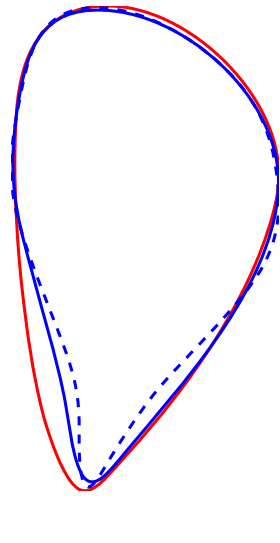

e)

Fig. 1 Poloidal flux level lines for free boundary snowflake equilibria: (a) fit to the shot \#36151 boundary $\delta_{u p}=0.2$; (b) increased upper triangularity $\delta_{u p}=0.4$, magenta dots show the target plasma shape; (c) large snowflake point triangularity $\delta_{\text {down }}=0.8$; (d) snowflake on the floor of the TCV vacuum vessel; (e) snowflake boundary from Fig.1b (blue) compared to the x-point boundary (red) and to the snowflake boundary with large pedestal current density (dashed). (Online colour: www.cppjournal.org).

\subsection{Pedestal profiles}

The plasma pressure profiles used in the study are based on the experimentally measured temperature and density profiles in TCV tokamak H-mode discharges [8]. The current density in the pedestal $\left(s>0.95\right.$, where $s=\bar{\psi}^{1 / 2}$ and $\bar{\psi}$ is normalized poloidal flux changing from 0 at the magnetic axis to 1 at the plasma boundary) was adjusted to be aligned with the collisionless bootstrap current. For sensitivity studies the pressure gradient profile $d p / d \psi$ in the pedestal was prescribed to be proportional to the derivative of the standard hyperbolic tangent (tanh) fit [9] 
for the pressure:

$$
\frac{d p}{d \psi} \sim 1-\tanh ^{2}\left(\frac{s_{0}-s}{w}\right),
$$

with the maximum attained at $s=s_{0}$. The experimental pedestal pressure profile shape is well described by $s_{0}=0.9865, w=0.015$. The pedestal profiles are added to the experimentally measured core profiles unchanged in the analysis. For the sensitivity study both the parallel current density profile $\left.(<j \cdot B\rangle_{\psi} /<R_{0} B \cdot \nabla \phi\right\rangle_{\psi}$, $<\cdot>_{\psi}=\frac{d}{d \psi} \int_{V_{\psi}} \cdot d V / \frac{d}{d \psi} V_{\psi}$ is the flux surface average over the volume between magnetic surfaces) and $d p / d \psi$ in the pedestal region are independently re-scaled to get the stability boundaries for the modes with different toroidal wave numbers $n$. The half-width $w$ and the location of the pedestal maximum $s_{0}$ are independent parameters in the study. The shift of the maximum location inside the plasma (decreasing $s_{0}$ ) is accompanied by a decrease in the ratio of the pressure gradient at the plasma boundary to its maximum value. In a recent predictive model for the pedestal height [10] this ratio was essentially fixed and only limited variations due to the uncertainty in the plasma edge determination are allowed. Different assumptions were made in [10] about the profile specifications in the pedestal, resulting in a ratio $p_{\text {egde }}^{\prime} / p_{\text {max }}^{\prime} \approx 0.1$. Fig. $2 \mathrm{~b}$ shows, however, that the family of profiles used in the present paper can well match the profiles used in [10] for a particular parameter setting.
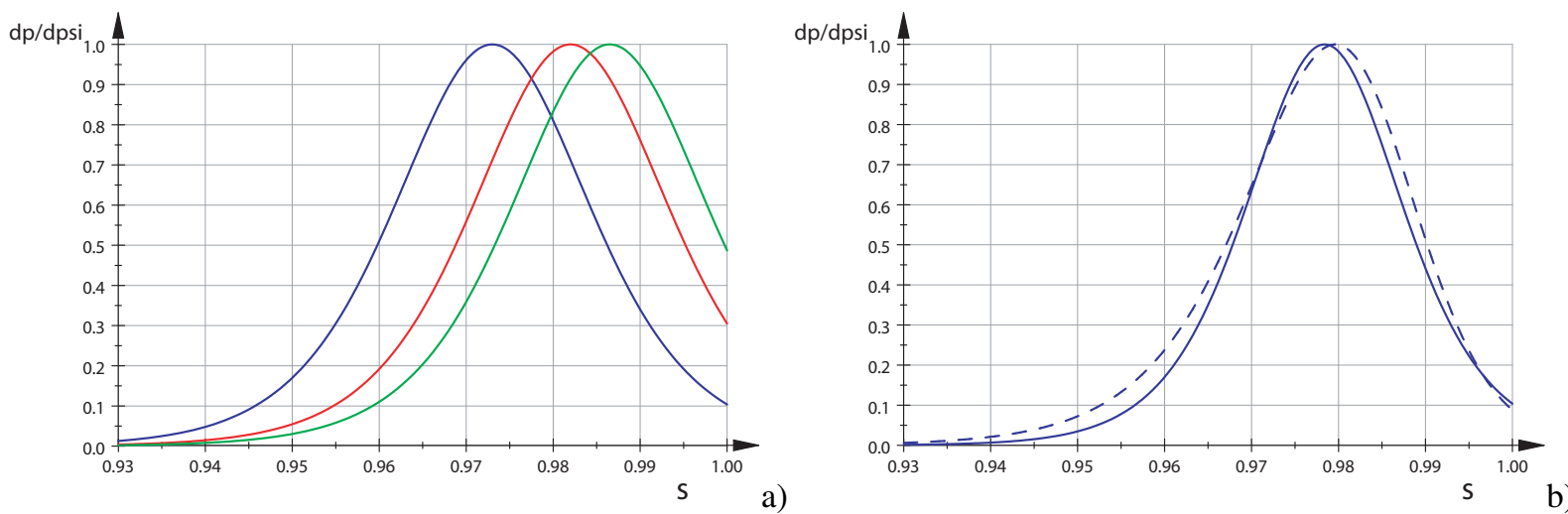

Fig. 2 The profiles of $d p / d \psi$ : (a) with different position of the maximum $s_{0}=1-1.8 w, 1-1.2 w, 1-0.9 w, w=0.015$; (b) approximation of the pressure gradient profile used in [10] with the width $\Delta=0.06$ (dashed line) by the $w=0.8 \Delta / 4, s_{0}=$ $1-1.8 w$ (solid line). Note that the corresponding pedestal depth $D=1-\left(s_{0}-w\right)^{2} \approx 1.11 \Delta$ is close to $\Delta$. (Online colour: www.cppjournal.org).

\subsection{Edge stability}

Four plasma shapes obtained in the free boundary calculations - SF and SN with low $(\delta=0.2)$ and high $(\delta=0.4)$ upper triangularity - were used in the series of fixed boundary equilibria for the edge stability diagram generation and pedestal profile sensitivity studies. The KINX code [11] was used for the stability calculations and the poloidal field null was assumed to be at the plasma boundary leading to a complete stabilization of localized peeling modes $[6,12]$.

The edge stability diagrams for SF and SN equilibria with the triangularity $\delta=0.4$ are compared in Fig.3. The presence of the second order null leads to an enhancement of the maximum attainable normalized pressure gradient $\alpha=2 \mu_{0} d p / d \psi d V / d \psi \sqrt{V /\left(2 \pi^{2} R_{0}\right)} /\left(4 \pi^{2}\right)$ [9] measured at the position of the maximum pressure gradient in the pedestal. Larger edge shear in the snowflake equilibrium results in a monotonic $q$ profile maintained at larger values of the parallel current density $J_{\|}=\max _{\text {pedestal }}<j \cdot B>_{\psi} /<|B|>_{\psi}$ (normalized by the crosssection area averaged current density $\langle J\rangle=I_{p} / S_{p}$ ) in the pedestal, i.e. a shear reversal in the pedestal takes place for larger values of $J_{\|} /\langle J\rangle$. However the current driven modes set the stability limit at approximately the same value of $J_{\|} /<J>\sim 1$. The enhancement in the maximum stable $\alpha$ for the SF is comparable to the 
same effect when increasing the triangularity from $\delta=0.2$ to 0.4 in the SN equilibrium. The stabilizing effects of SF and higher triangularity roughly "add up" in terms of maximum stable pressure gradient.

The normalized pressure gradient $\alpha$ reduces to $\alpha=-2 \mu_{0} \frac{R q^{2}}{B^{2}} d p / d r$ for large aspect ratio circular crosssection plasma that demonstrates its dependence on the value of $q$ at the position of the maximum in the pedestal. Due to high shear at the plasma edge it leads to significant difference in the values of $\alpha$ for the same pedestal pressure gradient. For the same reason it would also vary if the profiles in the pedestal are kept self-similar but the width changes. A good choice of an integral measure for the pressure gradient would be $\beta_{\text {ped }}=2 \mu_{0} p_{p e d} / B_{p, s x}^{2}$ [9], where $B_{p, s x}=\mu_{0} I_{p} / L_{p}$ is an averaged poloidal magnetic field at the separatrix with perimeter $L_{p}$ in the poloidal cross-section.
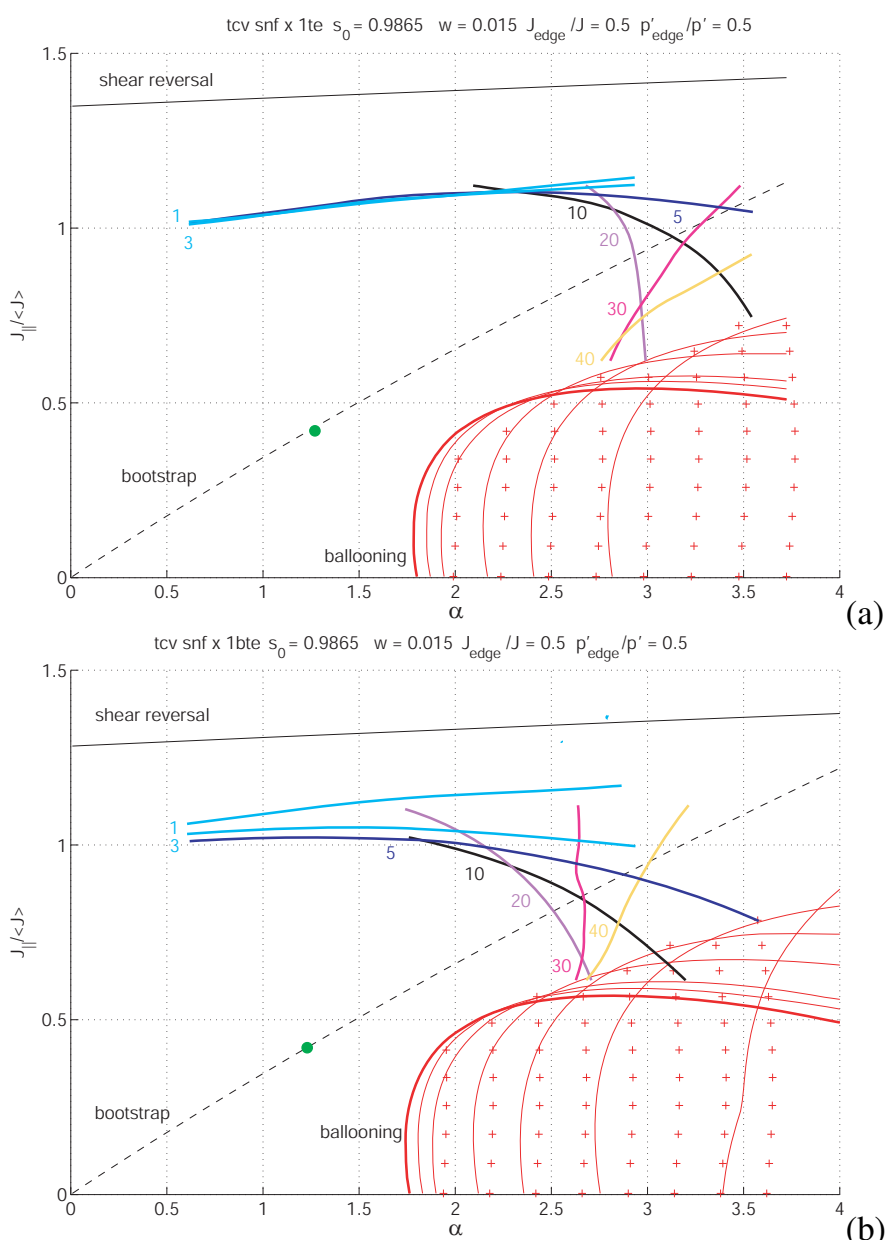

Fig. 3 Comparison of the edge stability diagrams for snowflake (a) and x-point (b) equilibria(the shapes shown in blue and red in Fig.1e respectively). The crosses and red lines show the high$\mathrm{n}$ ballooning mode stability boundaries. The shear is reversed above the solid black line. The dashed black line shows the collision-less bootstrap current density in the pedestal. Colored lines give the stability boundaries for medium $n=5-$ 40 kink-ballooning modes (toroidal wave numbers are shown). Light blue lines show the stability boundaries for the global modes $n=1,3$. The green circle corresponds to the pedestal parameters for the reference equilibrium. (Online colour: (b) www.cppjournal.org).

On the contrary to the pressure limit, the normalized current density at the pedestal maximum seems to be a suitable parameter for the edge stability diagram because the value of shear is directly related to it. For large aspect ratio, circular cross section plasma $J_{\|} /<J>=(2-S) / 2$, where $S=(r / q) d q / d r$ is the shear. The current limit with separatrix at the boundary appears to have a close relation to the instability condition $J_{\|} /\langle J\rangle>1$ [13] for the external kink mode with toroidal and poloidal wave numbers $n$ and $m$ in a circular plasma with $m-n q=1$ at the boundary, i.e. when the resonant surface in vacuum is far from the plasma. Thus for a large aspect ratio circular plasma the shear reversal and the normalized current density $\left.J_{\|} /<J\right\rangle=1$ limits coincide. Shaping of the plasma cross section, in particular triangularity, increases the threshold value of $J_{\|} /\langle J\rangle$ necessary to reach the shear reversal. The condition $J_{\|} /\langle J\rangle=1$ does not exactly correspond to the stability and shear reversal limits in a shaped toroidal plasma. However, for $J_{\|} /\langle J\rangle>1$ in the pedestal increasingly flat and non-monotonic safety factor profile eventually leads to coupling of low-n external kink 
modes to global interchange- or infernal-type modes and the current driven destabilization. Moreover, resistive mode stability and associated magnetic field reconnection with multiple resonant surfaces can come into play.

Unlike the Troyon scaling for the toroidal beta limit against pressure driven external kink modes which essentially depend on the normalized plasma current and toroidal field, the edge kink-ballooning pressure limit can be well described in terms of poloidal beta with toroidal field entering only through the q-values in the pedestal. The reason for that is connected with the $w \times n \times q_{95}=$ const scaling proposed in [6] implying that a change in $q$ is compensated by adjusting the toroidal wave number $n$ to have approximately the same number of rational surfaces in the pedestal region for a given value of shear, i.e. for a specific point in an edge stability diagram. On the other hand, changes in $q$ lead to changes in the $n=\infty$ ballooning mode behavior that in turn affects the stability limits against coupled peeling/ballooning modes. However this effect can be taken into account by normalizing the pressure gradient to its ballooning mode limit at the separatrix [6] or explicitly introducing the normalized current into the scaling for poloidal beta.
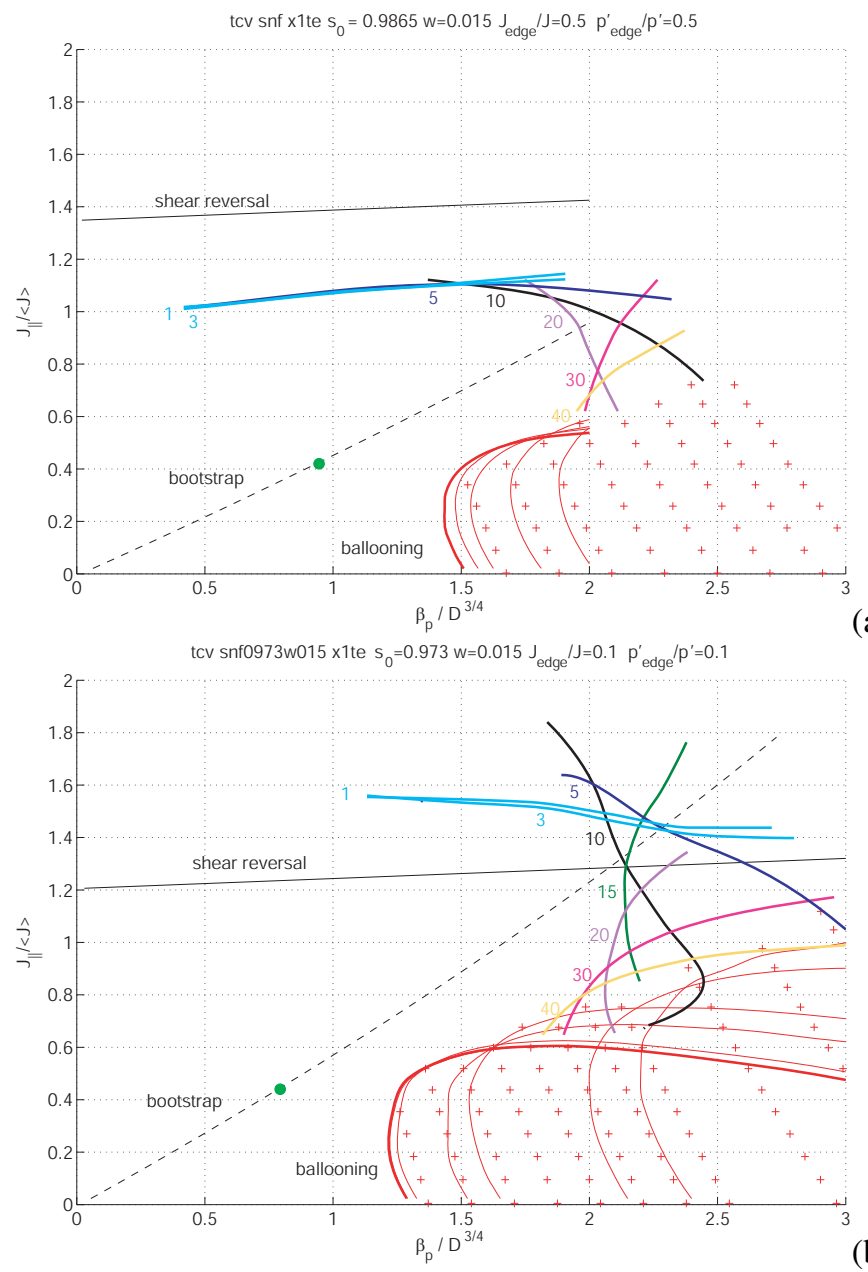

Fig. 4 Edge stability diagrams for SF with different ratio of the edge to maximum values in pedestal, $w=0.015$ : (a) $s_{0}=1-0.9 w, p_{\text {edge }}^{\prime} / p_{\max }^{\prime}=0.5$; (b) $s_{0}=1-1.8 w, p_{\text {edge }}^{\prime} / p_{\max }^{\prime}=0.1$. (Online (b) colour: www.cppjournal.org).

Estimating the width of the pedestal under given experimental conditions is a separate and complicated task. However, the scaling for the pedestal poloidal beta is certainly related to an averaged pressure gradient in the pedestal and a suitable definition of the pedestal width should be chosen to better represent the stability limits. For the standard tanh fit the distance $D$ from the edge $(s=1)$ to the knee of the pedestal $\left(s=s_{0}-w\right)$ measured in units of the normalized poloidal flux $D=1-\left(s_{0}-w\right)^{2}$ (further referred to as pedestal depth) seems to be an appropriate parameter for the scaling in the form:

$$
\beta_{p, p e d}=C D^{\gamma}
$$


where the pedestal pressure $p_{\text {ped }}$ is estimated at the knee of the pedestal and $\gamma$ is close to 1 . As concerns the exponent $\gamma$ deviation from unity, both change of the pedestal profile shape (location of the maximum, in particular) and the width of the pedestal lead to changes in the ballooning mode stability related to the shear reversal and the second stability access. For the family of profiles (1) a shift of the maximum deeper into plasma is accompanied by a decrease of edge/maximum ratio, which is stabilizing for current driven modes. To some extent the corresponding increase of the limiting pressure can be compensated by an increase of the pedestal depth due to smaller $s_{0}$ leaving the scaling coefficient $C$ approximately constant for some exponent $\gamma$. The deviation of $\gamma$ from unity provides a measure of the combined influence of the pedestal profile change on the ballooning and external mode stability. In turn such an influence depends on the plasma shaping, for example at larger triangularity the second stability access takes place for lower current density (higher shear) and for lower toroidal mode numbers. For fixed pedestal profile shape the dependence on the pedestal width can be approximated by $\gamma=0.75$ [14]. Two explanations were proposed for the fact that $\gamma$ deviates from unity, i.e. the pedestal stability limit is not strictly a limit on the pressure gradient: (1) finite-n modes are non-local and are directly sensitive to the pedestal width as well as local parameters, and (2) the natural magnetic shear in the middle of the pedestal, where gradients are steepest, decreases with pedestal width. The second explanation implies that the plasma shaping also plays a role. In fact, the deviation was not recognized in the TCV edge stability studies [6] performed for low triangularity SN plasmas for which $\gamma$ is closer to unity.
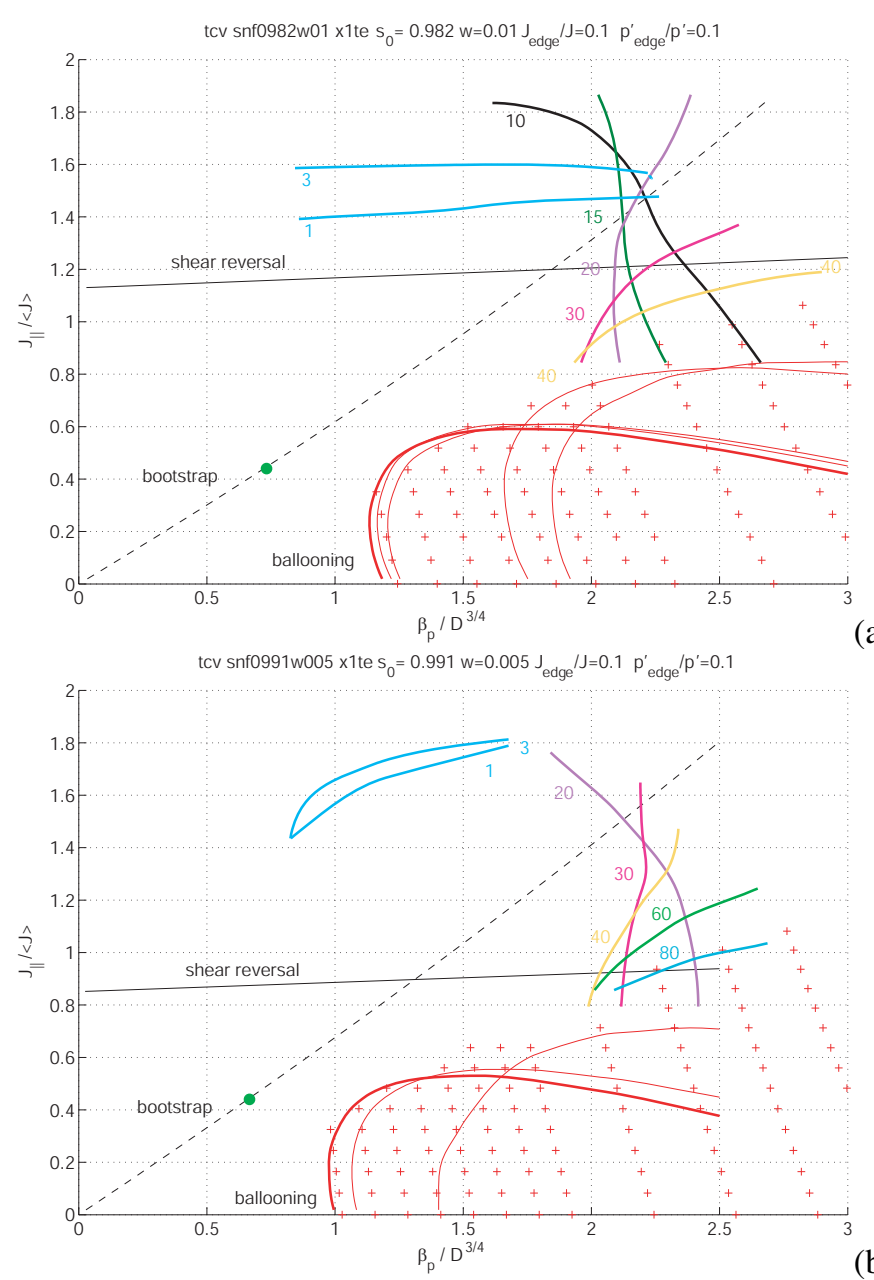

Fig. 5 Edge stability diagrams for SF with different pedestal width but fixed shape $s_{0}=1-1.8 w$, $p_{\text {edge }}^{\prime} / p_{\text {max }}^{\prime}=0.1:$ (a) $w=0.01$; (b) $w=0.005$. (b) (Online colour: www.cppjournal.org).

For the SF plasma with upper triangularity $\delta=0.4$ the value of $\gamma=0.75$ in (2) fits the $\beta_{p, p e d}$ under variations of the pedestal half-width $w$ with fixed pedestal profile shape $s_{0}=1-c w, c=$ const and $\gamma=0.85-$ under variations of $s_{0}$ together with the pedestal depth $D=1-\left(s_{0}-w\right)^{2}$ when $w$ is fixed. Fig.4 presents the stability 
diagrams for different shapes of the pedestal profile in the parameter plane $\left(\beta_{p, p e d} / D^{0.75}, J_{\|} /<J>\right)$. In Fig.5 the stability diagrams for different values of the pedestal half-width $w$ are compared.

\section{Conclusions}

The edge stability of snowflake equilibria is enhanced compared to a standard SN divertor plasma especially in a combination with increased upper triangularity: the values of the pedestal poloidal beta that can be reached in the TCV SF configurations increases from $C=\beta_{p \text {,ped }} / D^{0.75}=1.8-1.9$ to $2.1-2.2$, where $D$ is the depth of the pedestal measured from plasma edge to the pedestal knee. Combined with the scaling for the pedestal depth $D=0.076 \beta_{p, p e d}^{0.5}[10], C=2.2$ corresponds to a predicted depth $D=0.03$ that is twice as narrow compared to the measured pedestal profiles in TCV [8]. For the TCV parameters $\left(B=1.4 T, a=0.22 \mathrm{~m}, I_{p}=0.3 M A, I_{N}=\right.$ $\left.I_{p} /(a B)=0.97, n_{e, p e d}=3 \cdot 10^{19} \mathrm{~m}^{-3}\right)$ and assuming $T_{i}=T_{e}$ it gives pedestal temperature $T_{e}=250 \mathrm{eV}$ and $\beta_{N, \text { ped }}=2 \mu_{0} p /\left(B^{2} I_{N}\right)=0.32$.

The $\beta_{p, p e d}=C D^{0.75}$ scaling provides a reasonable approximation for the pressure limited by the kinkballooning modes under variation of the pedestal width and profile shape, at least within the tanh fit profile family. The scaling should be modified to explicitly take into account the enhancement of the stability limits against coupled kink-ballooning modes due to better ballooning mode stability for lower normalized plasma current (in particular, for higher $q$ deeper magnetic well corresponds to higher pressure gradients in the first region of ballooning mode stability). In [6] this effect was taken into account by normalization to limiting pressure gradient at the separatrix.

The ratio of the edge current density to the maximum in the pedestal is an important parameter that qualitatively changes the edge stability. Even if the scaling $\beta_{p, p e d}=C D^{0.75}$ for the overall pressure limit holds under variations of pedestal profile shape, provided that the pedestal depth $D$ is appropriately defined, the stability diagrams change with respect to the location of the stability boundaries versus the shear reversal and the region accessible for bootstrap current aligned equilibria. In particular, for low edge current densities the maximum pressure is reached with reversed shear in the pedestal, the limiting toroidal wave numbers are lower for the same pedestal width and $n=1$ mode sets the limit against current driven modes.

Acknowledgements This work was partly supported by the Swiss National Science Foundation.

\section{References}

[1] F. Piras et al. Plasma Phys. Control. Fusion 51055009 (2009).

[2] S. Yu. Medvedev et al. Proceedings 36th EPS Conference on Plasma Physics, Sofia, Bulgaria, Volume 33 (Europhysics Conference Abstracts, 2009), P2.143.

[3] D. D. Ryutov et al. Phys. Plasmas 14064502 (2007).

[4] D. D. Ryutov et al. Phys. Plasmas 15092501 (2008).

[5] P. B. Snyder, H. R. Wilson et al. Phys. Plasmas 92037 (2002).

[6] S. Yu. Medvedev et al. Plasma Phys. Control. Fusion 48927 (2006).

[7] A. A. Ivanov, R. R. Khayrutdinov, S. Yu. Medvedev, Yu. Yu. Poshekhonov, Preprint, Keldysh Inst. Appl. Math., the Russian Academy of Sciences, \#39 (2009). http://www.keldysh.ru/papers/2009/source/prep2009_39.doc

[8] R. Behn et al. Plasma Phys. Control. Fusion 49128 (2007).

[9] R. J. Groebner and T. H. Osborne Phys. Plasmas 51800 (1998).

[10] P. B. Snyder et al. Physics of Plasmas 16056118 (2009).

[11] L. Degtyarev et al. Comput. Phys. Commun. 10310 (1997).

[12] G. T. A. Huysmans, Plasma Phys. Control. Fusion 472107 (2005).

[13] J. W. Connor et al. Physics of Plasmas 52687 (1998).

[14] P. B. Snyder et al. Plasma Phys. Control. Fusion 46 A131 (2004). 